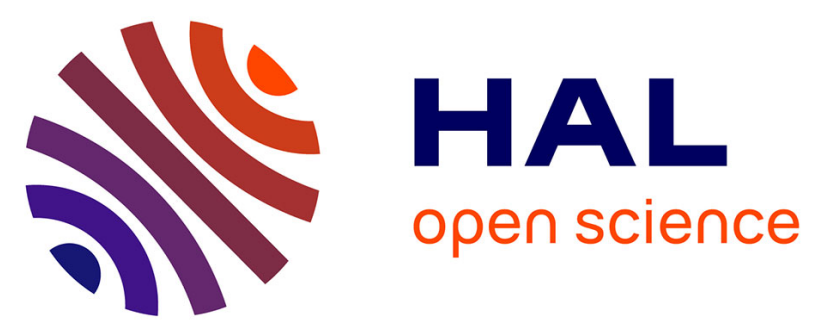

\title{
Home health care daily planning considering the satisfaction of all the stakeholders
}

\author{
Liwen Zhang, Pierre-Yves Bouchet, Elyes Lamine, Franck Fontanili,
} Christophe Bortolaso, Musthapha Derras, Hervé Pingaud

\section{- To cite this version:}

Liwen Zhang, Pierre-Yves Bouchet, Elyes Lamine, Franck Fontanili, Christophe Bortolaso, et al.. Home health care daily planning considering the satisfaction of all the stakeholders. IESM 19 - 8th International Conference on Industrial Engineering and Systems Management, Sep 2019, Shangai, China. p.1-6, 10.1109/IESM45758.2019.8948201 . hal-02415431

\section{HAL Id: hal-02415431 https://imt-mines-albi.hal.science/hal-02415431}

Submitted on 12 Mar 2020

HAL is a multi-disciplinary open access archive for the deposit and dissemination of scientific research documents, whether they are published or not. The documents may come from teaching and research institutions in France or abroad, or from public or private research centers.
L'archive ouverte pluridisciplinaire HAL, est destinée au dépôt et à la diffusion de documents scientifiques de niveau recherche, publiés ou non, émanant des établissements d'enseignement et de recherche français ou étrangers, des laboratoires publics ou privés. 


\section{Home Health Care Daily Planning Considering the Satisfaction of all the Stakeholders}

\author{
Liwen Zhang \\ DRI and Industrial Engineering Centre \\ Berger-Levrault and IMT Mines Albi \\ Labège, France and Albi, France \\ liwen.zhang@berger-levrault.com
}

\author{
Christophe Bortolaso, Mustapha Derras \\ DRI \\ Berger-Levrault \\ Labège, France \\ \{first name.last name\} @ berger-levrault.com
}

\author{
Pierre-Yves Bouchet, Elyes Lamine, Franck Fontanili \\ Industrial Engineering Centre \\ University of Toulouse-IMT Mines Albi \\ Albi, France \\ \{first name.last name\}@ mines-albi.fr
}

\author{
Hervé Pingaud \\ CNRS LGC \\ University of Toulouse-INU Champollion \\ Albi, France \\ herve.pingaud@univ-jfc.fr
}

\begin{abstract}
Nowadays, the aging population and the growth of life expectancy lead to an increase of people experiencing frailty and therefore loss of autonomy. As there are not enough places available in specialized institutions, an alternative is Home Health Care (HHC) services. The HHC structure is willing to optimize the scheduling of the requested care services by satisfying the requirements of patients, the preferences of caregivers, and by balancing the workload among the different caregivers. The purpose of this paper is to propose a metaheuristic-oriented formulation and a simulated annealing algorithm for generating an optimal short-term (daily) routing and scheduling solution to reach maximum satisfaction among stakeholders (patients and caregivers) in an HHC system. With this method computed on a real-world dataset, we obtained satisfying scheduling solutions.
\end{abstract}

Keywords - Home Health Care, Short-term scheduling and routing, Metaheuristic formulation, Simulated annealing.

\section{INTRODUCTION}

The aging population and increasing life expectancy in France and in other developed countries leads to an increasing number of elderly people suffering with loss of autonomy and in danger due to frailty. Those patients usually suffer from chronic diseases which require long-term care. They largely prefer to stay at home rather than living longer in medicalized institutions such as hospitals. That is why we are currently witnessing the rapid growth of Home Health Care (HHC) structures. In addition, due to the evolution of the public health code in accordance with the health law of 2016 in France, society is aware of the necessity to associate the beneficiaries of care services in a coordinated care path. This requires continuity and better synergy between caregivers. However, the compliance of practices in the field is slow to develop in regard with these institutional guidelines. The consideration of multiple criteria, especially in the different views of stakeholders (patients and caregivers) in HHC systems, makes it more difficult to schedule the caregivers because they need to respond efficiently the patients' requirements in the short-term.

Considering this context, there is a need for a system able to manage home-based social, medical and medical-social care services, as well as paramedical professionals around the patients, mentioned in a recent work [1]. As a result, planning generation should take into account the satisfaction of each stakeholder, which is a key to ensuring efficient and operational coordination in HHC. Therefore, comprehensive and multidisciplinary planning provides the ability to transmit fluid, high quality date to all the stakeholders to guarantee the satisfaction of both patients and caregivers. Furthermore, the structure is used to optimize the scheduling with respect to the patients' requirements, the caregivers' preferences and balance the workload among various caregivers. Thus, throughout this paper, the research takes the stakeholders' satisfaction as a decisive component to process daily planning using a system based on a metaheuristic-oriented mathematical formulation. We also study performance of a simulated annealing resolution algorithm to demonstrate the viability of the resulting schedule and overall staff satisfaction.

The following content is divided into five parts. The related work is presented in section 2. We review short-term scheduling and routing techniques dedicated to HHC. In section 3 , we present the context of the problem that needs to be resolved. Next, we provide a general overview of the obtained results with a simulated annealing algorithm and we study its performance on real world data. The last section announces remarks and our outlooks.

\section{RELATED WORK}

In recent years, Home Health Care Routing and Scheduling Problems (HHCRSP) have been studied extensively. Recent work [2][3] shows issues when considering different planning horizons for the HHCRSP. This review takes a short-term planning horizon. We also focus on enumeration of the similar constraints, the objective functions and possible resolutions approaches, which are enumerated in recent literature.

Mankowska et al. [4] dealt with the coordination through a daily planning model of care services performed by HHC staffs. En-nahli et al. [5] presented a study aimed at finding feasible work schedules for each resource. It is based on a daily horizon to ensure patients' and caregivers' satisfaction by controlling the routing costs and respecting patients' preferences. For the most detailed studies, several insights have been learned by 
analyzing this synthesis bibliographically in Table I. It is appropriate to discuss the perspectives related to the decision. We choose the total satisfaction of patients as the reference to achieve the patients' expected service. This is a form of balance between offer and demand which is reflected in the HHCRSP. However, the short time horizons play a key role, both days and weeks are the preferred time intervals. Furthermore, a list of criteria used in the formulation of the HHC is also presented. For the caregivers the following criteria are considered: the qualification (Quali. C), the availability (Avail. C), the frequency of assignment according to the qualification (Freq. C), the maximum working hours (Work h. C), the break (Break C), the preferred schedules (Pref. sc. C), the different living places (Live p. C). As for the patients the following criteria are considered: the availability (Avail. P), the interdependences among the requested care services (Inter. P), the special needs (Need. P).

TABLE I. THE CRITERIA CONSIDERED IN SHORT-TERM HHRSP

\begin{tabular}{|c|c|c|c|c|c|c|c|c|c|c|c|c|c|c|}
\hline \multirow[t]{2}{*}{ Ref. } & \multicolumn{2}{|c|}{ View } & \multicolumn{2}{|c|}{ Horizon } & \multicolumn{10}{|c|}{ Constraints } \\
\hline & 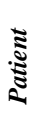 & 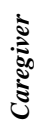 & 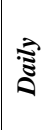 & $\begin{array}{l}\overrightarrow{2} \\
\frac{2}{2} \\
\vdots\end{array}$ & 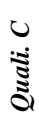 & 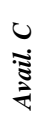 & نे & 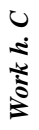 & $\frac{u}{3}$ & $\begin{array}{l}u \\
\dot{0} \\
\dot{0} \\
\dot{0} \\
\dot{0}\end{array}$ & 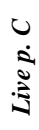 & 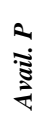 & $\begin{array}{l}2 \\
\vdots \\
\Xi \\
\Xi\end{array}$ & $\frac{2}{8}$ \\
\hline $\begin{array}{r}\text { Mankowska } \\
\text { and al [4] }\end{array}$ & $X$ & & $X$ & & $X$ & $X$ & & & & & & $\mathrm{X}$ & $X$ & $\mathrm{X}$ \\
\hline $\begin{array}{r}\text { Trautsamwieser } \\
\text { and Hirsch[6] }\end{array}$ & & $X$ & & $\mathrm{X}$ & $X$ & $\mathrm{X}$ & $X$ & & & & & $\mathrm{X}$ & & \\
\hline $\begin{array}{r}\text { En-nahli and } \\
\text { al.[5] }\end{array}$ & $\mathrm{X}$ & & $X$ & & $X$ & $X$ & & & & & & $\mathrm{X}$ & & $\mathrm{X}$ \\
\hline $\begin{array}{r}\text { Yuan and } \\
\text { Fügenschuh [7] }\end{array}$ & & $X$ & & $X$ & $\mathrm{X}$ & $X$ & & & & $X$ & & $\mathrm{X}$ & & $\mathrm{X}$ \\
\hline Shao and al. [8] & & $X$ & $X$ & $X$ & $X$ & $X$ & $X$ & $X$ & $X$ & & & $X$ & & \\
\hline $\begin{array}{r}\text { Hiermann and } \\
\text { al. [9] }\end{array}$ & & $X$ & $X$ & $\mathrm{X}$ & $\mathrm{X}$ & $X$ & & $X$ & & $X$ & & $\mathrm{X}$ & & \\
\hline $\begin{array}{r}\text { Redjem and } \\
\text { Marcon [10] }\end{array}$ & & $X$ & $X$ & $\mathrm{X}$ & $\mathrm{X}$ & $\mathrm{X}$ & & & & & & $\mathrm{X}$ & & $\mathrm{X}$ \\
\hline Du and al.[11] & & $\mathrm{X}$ & $X$ & $\mathrm{X}$ & $\mathrm{X}$ & $\mathrm{X}$ & & & & & $X$ & $X$ & & $\mathrm{X}$ \\
\hline
\end{tabular}

Depending on the mathematical nature of the variables characterizing the decisions, the solutions vary according to one or more criteria including the types of offer and the demand constraints that are allowed in the mathematical formulation of the HHCRSP. There are three group resolution approaches: (i) exact method based on Integer Linear Programming (ILP) or Mixed Integer Linear Programming (MILP), (ii) approximated method based on a metaheuristic algorithm and (iii) hybrid methods combining the first two methods.

For the exact method, Yuan et al. [12] present a stochastic programming model by using a branch and bound, label and column generation algorithm. En-nahli et al. [5] introduce a multi-objective approach based on MILP, a multi-objective modeling with weighted sum and a heuristic, considering the problem with multi-objective's character to assign caregivers which respect patient's satisfaction. For the approximated method, Rest and Hirsch [13] propose an approach with Tabu research for calculating the travel times depending on the schedules of public transport. Finally, for the hybrid method, Yuan and Fügenschuh [7] formulate the problem as ILP and also develop a greedy algorithm and local search approaches to optimize their results. The recent research of Martinez et al. [14] generate a MILP model to plan the daily care services in one week. The initial solution of this work is generated by the greedy algorithm.
Through the review of the related works, we observe a lack of consideration of patient and the caregiver satisfaction. However, for the resolution approach, the exact method costs much more computing time than the approximated method (i.e. heuristic, metaheuristic algorithm) to solve the HHCRSP optimization (NP-hard problem). Consequently, as the satisfaction of all the stakeholders is our first and key area of study, in this paper, we will describe a mathematical formulation using the approximated method to solve this problem within a reasonable computational time.

\section{PROBLEM DESCRIPTION}

\section{A. Problem statement}

The main objective of our work is to help HHC structure in organizing its employees' planning by satisfying the requirements of the registered patients and maximize the satisfaction of both patients and caregivers during one service day. On one hand, the patients' satisfaction implies the respect of the requested time window per care service and of the requested delay between two successive care services. On the other hand, caregiver satisfaction implies respect for working hours and balancing the difficulties of the performed care. However, in a daily scheduling situation, the HHC structure has several caregivers with various skills and abilities which must be aligned with patient demands for given time windows. The HHC system is organized in three periods a day (i.e. morning, afternoon and evening), which categorize the working caregivers by each period of the day according to their availabilities.

The caregivers are characterized by their ID, their professional type, their skills list and several specific characteristics including working time windows per period and the tolerance of working overtime. The performed care services during a specific period by one caregiver is defined as a round. If a caregiver works multiple periods per day, each of his performed care services during one period is considered as an independent round. In other words, a caregiver can execute several independent rounds per day.

To characterize the patients, we need his residence address, his minimum intraoperative delay between two requested care services, the non-respect tolerance of the expected appointment hours and the tolerance regarding the interoperative delay.

To characterize the care services, we need the ID, the care act name, the performance duration, the required skills and the difficulty level measured by the GIR score. GIR is the abbreviation of "Iso-Resource Group" in French, presenting the level of an elderly person's loss of autonomy. GIR $=1$ is the highest level of loss of autonomy, while GIR=6 is the lowest. To clarify, if a patient demands two different care services, the GIR remains the same regardless of the quantity of requests.

\section{B. Hypothesis}

In this section, we present the assumptions considered for generating a daily scheduling and routing.

- All caregivers start theirs rounds at the beginning time announced on their schedules.

- The rounds performed by each caregiver without breaks: the caregiver with respect to the "transit - execution of care service - transit" until the end of the day. 
- The length of the rounds (i.e. the distances travelled) is not considered. Under our assumption, all transit between the patients' residence is regarded as a bird'seye view. Knowing the average speed of a vehicle and the GPS coordinates of the residence, we can then compute the travel time.

- A round starts and finishes at the HHC structure. All travels between departure and arrival are considered as the working time of each caregiver: the last round of care service should not be accomplished too late otherwise the satisfaction of the caregiver will decline, which will result in an excess of overtime worked.

- Care services requiring two or more caregivers at the same time are not considered.

- The stakeholders who are out of service on a given day due to illness or vacation are not considered.

\section{Businesses oriented constraint enumeration}

To calculate a satisfying scheduling and routing result, we must first understand the constraints which limit the propositions. The solutions cannot be relevant if we don't follow real businesses-oriented constraints. Two types of constraints are taken into consideration: 1) strong constraints, which must absolutely be respected and 2) weak constraints that may be violated but the more they are respected the better the participants' satisfaction levels.

\section{1) Strong businesses-oriented constraints:}

a) A caregiver should only perform the care services for which he or she is qualified.

b) A care service cannot be assigned to a caregiver lacking sufficient skills.

c) A patient who requires a specific caregiver can only be treated by the designated caregiver.

d) No care service can be aborted: an unperformed care service in one day is not acceptable.

2) Weak business-oriented constraints

e) The difficulty of all the performed rounds should to be balanced. The difficulty for one round is the average difficulty of the performed care services in this round regarding all those scheduled.

f) The duration of a round for a caregiver should be within the given working time, otherwise, the overtime should be under the limit of tolerance which is required by the caregiver.

g) The request of the patient should be processed on time or the overtime must be within their tolerance.

h) The inter-operative delay between two successive care services requested by patients should be maximized.

\section{MATHMATICAL FORMULATION}

In this section, the presentation starts with the explanation of the parameters and variables. We then detail the objective function components which consist of the business-oriented constraint. Finally, our objective function will be presented by assembling all the components.

\section{A. Parameters}

$P$ : Set of patients.

$S$ : Set of care services.

$C:$ Set of available caregivers in the HHC structure.

$H$ : Set of opening periods for HHC structure.

$P E(c)$ : Set of working periods of caregiver $c$.

$S(p e)$ : Set of requested care services per opening period $p e \in H$ of $\mathrm{HHC}$ structure.

$S(p)$ : Set of care services requested by patient $p$.

$v$ : Total number of care services requested per day.

$I(s, c)$ : Equal to 1 if caregiver $c$ imposed by care service $s$ requested by one patient, 0 otherwise.

$\operatorname{ped}(s)$ : Requested period in one day of the care service $s$.

$[\alpha(s), \beta(s)]$ : Time windows requested by patient for the care service $s$

$\operatorname{gir}(s)$ : Difficulty of providing a care service $s$.

type $(s)$ : Profession type required by care service $s$.

skills(s): Skills required by care service $s$.

duration $(s)$ : Duration of care service $s$.

$\operatorname{travel}\left(s_{1}, s_{2}\right)$ : Travel time between service $s_{1}$ and $s_{2}$

$\operatorname{cts}(p)$ : Tolerance for overtime of the care services requested by patient $p$.

$r s p(p)$ : Rate of a patient's satisfaction regarding the care service requested

by $p$ having $\operatorname{cts}(p)$ minutes' excess of the time window's beginning.

$\operatorname{delay}\left(s_{k}, s_{k+1}, p\right)$ : Inter-operative delay between two successive care services

$s_{k}, s_{k+1}$ requested by patient $p$

$\operatorname{cit}\left(s_{k}, s_{k+1}, p\right)$ : Tolerance for non-respect of $\operatorname{delay}\left(s_{k}, s_{k+1}, p\right)$ per patient $\mathrm{p}$.

$r s i\left(s_{k}, s_{k+1}, p\right)$ : Rate of a care patient's satisfaction, with a lack of $\operatorname{cit}\left(s_{k}, s_{k+1}, p\right)$ minutes to $\operatorname{delay}\left(s_{k}, s_{k+1}, p\right)$

$\operatorname{skills}(c)$ : Skills of caregiver $c$.

type $(c)$ : Professional type of caregiver $c$

$[a(c, p e), b(c, p e)]$ : Working time windows for caregiver $c$ per working period $p e \in H$. (i.e. the working TW for caregiver $c$ in the $p e=$ "morning" is [8:00-10:00].)

ct $(c)$ : Tolerance for the overrun of the working time windows requested by caregiver per period in one day, beyond which the caregiver $c$ is no longer satisfied with the round imposed.

$r s(c)$ : Rate of a caregiver's satisfaction regarding the round having $\operatorname{ct}(c)$ minutes' overrun of the working time windows.

\section{B. Variables}

$G(s, c)$ : Equal to 1 if caregiver $c$ qualified to provide care service s requested by one patient, 0 otherwise.

overtime $(s)$ : Overtime between the time window requested by patient and the real beginning time for care service $s$

$\operatorname{start}(s)$ : Start time of care service $s$.

$\operatorname{timelack}\left(s_{k}, s_{k+1}, p\right)$ : Compared with the $\operatorname{delay}\left(s_{k}, s_{k+1}, p\right)$, the lacking inter-operative time between care service $s_{k}, s_{k+1}$ requested by patient $p$.

satisdelay $\left(s_{k}, s_{k+1}, p\right)$ : Satisfaction of the inter-operative delay between two successive care services for patient $p$.

$\operatorname{satiscc}(s, p)$ : Satisfaction of the respect of time windows of care service $s$ requested by patient $p$.

overtimecg $(c, p e)$ : Overtime between the working time window of caregiver $\mathrm{c}$ and the real working time per working period $p e$ of caregiver $c$.

finish $(c, p e)$ : Finishing time of caregiver $c$ per her working period $p e$ num $(c, p e)$ : Number of care services performed by caregiver $c$ in working period $p e$.

difficulty $(c, p e)$ : Difficulty of the set of care services performed by caregiver $\mathrm{c}$ within each of his working periods $p e$

$\operatorname{satiscg}(c, p e)$ : Satisfaction of the work load for caregiver $c$ within each of his working periods $p e$

\section{Component of the objective function}

In general, an objective function (OF) is an aggregation of the solution's evaluation in accordance with the different criteria. This section aims to present the OF components which integrate all the constraints that have been mentioned in section 3. Our OF is based on a function that seeks feasibility before the optimization. In another words, it must respect exactly all the strong constraints $a), b), c), d$ ). Moreover, as for the feasibility quantifying compliance with the strong constraints, the OF also assesses the level of compliance with the weak 
constraints $e), f), g$ ), $h$ ). This second evaluation is a kind of "optimization calculation". Its goal is to maximize the satisfaction of the weak constraints by respecting the satisfaction of the two sets of stakeholders.

\section{- Feasibility component}

There must be coherence between demand (professional types, the required caregiver's skills and specific impositions) and offer (professional types and skills owned by the caregivers). This component links with the strong constraints $a$ ) b) c) d).

$$
C(s, c)=\left\{\begin{array}{c}
1 \text { if type }(c)=\text { type }(s) \text { and skill }(c) \in \operatorname{skill}(s) \\
1 \text { if } I(s, c)=1 \\
0 \text { otherwise }
\end{array}\right.
$$

$\forall s \in S, \forall c \in C$ and $G(s, c)=\{0,1\}, I(s, c)=\{0,1\}$

\section{- Optimization component}

As for weak constraint e), this component (2) computes the difficulties around the set of performed care services by means of one caregiver within the work period. In brief, the difficulties of a round of each caregiver.

$\operatorname{difficulty}(c, p e)=\min \left(1, \frac{\frac{\sum_{s_{i} \in S(p e)} \operatorname{gir}\left(s_{i}\right) G\left(s_{i}, c\right)}{\operatorname{num}(c, p e)}}{\frac{\sum_{s \in S} \operatorname{gir}(s)}{v}}\right)$

$\forall c \in C, \forall p e \in P E(c)$ and difficulty $(c, p e) \in[0,1]$, gir $\in[1,6]$,

$$
v \geq 0, \operatorname{num}(c, p e) \geq 0
$$

The expression difficulty therefore refers to one for all rounds where the average GIR score of all the care services is higher than that of all care services to be carried out in one day. The value tends towards 0 when the round includes too many care services with a weak GIR score. The greater the value of difficulty, the easier the performance. Therefore, the rounds can be balanced by evaluating this function component in each round.

To formulate the weak constraint $f$ ), the first component is the first step calculated by (3) which is to figure out the overtime for one caregiver per working period. The second component (4) is the second step which is for calculating the satisfaction of the assigned work load for one caregiver within each working period

$$
\begin{aligned}
& \operatorname{overtimecg}(c, p e)=\max ((b(c, p e)-a(c, p e)) \\
& -(\operatorname{finish}(c, p e)-a(c, p e)), 0) \\
& \forall c \in C, \forall p e \in P E(c) \text { overtimecg }(c, p e) \geq 0, \text { finish }(c, p e) \\
& \geq 0, a(c, p e) b(c, p e) \in[0,1440] \\
& \operatorname{satiscg}(c, p e)=\frac{1}{1+\left(\frac{100}{r s(c)}-1\right)\left(\frac{\text { overtimecg }(c, p e)}{c t(c)}\right)^{2}} \\
& \forall c \in C, \forall p e \in P E(c) \text { and } \operatorname{satiscg}(c, p e) \in[0,1], \operatorname{ct}(c) \geq 0, r s(c) \\
& \in[0,50]
\end{aligned}
$$

A weak constraint $g$ ) is similar to $f$ ) except for patients. The importance is the comparison between the real start time and the time window imposed by the patient. The time window corresponds to the earliest and the latest start times of one care service. Thus, the scheduling time should ideally be within this range. To explain this constraint in a mathematical way, first, the overtime for each care service is computed by (5). Secondly, the precedence of the pair of care services is ensured by (6). Finally, the satisfaction regarding respect of time windows of each care service is computed by (7).

$$
\begin{gathered}
\operatorname{overtime}(s)=\max (\operatorname{start}(s)-\beta(s), \alpha(s)-\operatorname{start}(s), 0) \\
\operatorname{start}\left(s_{2}\right) \geq \operatorname{start}\left(s_{1}\right)+\operatorname{duration}\left(s_{1}\right)+\operatorname{travel}\left(s_{1}, s_{2}\right) \\
\forall s, s_{1}, s_{2} \in S \text { and overtime }(s) \geq 0, \operatorname{start}(s) \geq 0, \alpha(s) \beta(s) \\
\in[0,1440], \operatorname{travel}\left(s_{1}, s_{2}\right) \geq 0
\end{gathered}
$$

$$
\operatorname{satiscc}(s, p e)=\frac{1}{1+\left(\frac{100}{r s p(p)}-1\right)\left(\frac{\operatorname{overtime}(s)}{\operatorname{cts}(p)}\right)^{2}}
$$

$\forall p \in P, \forall s \in S(p)$ and $\operatorname{satiscc}(s, p e) \in[0,1], \operatorname{cts}(p) \geq 0, r s p(p)$

$$
\in[0,50]
$$

For weak constraint $h$ ), it is necessary to compare the delay between two successive care services performed by a caregiver with the requested interoperative delay of the patient. To be more precise, if the real delay is longer than the requested delay, the patient's satisfaction about the interoperative delay is maximum. Otherwise, the lack of interoperative delay will reduce patient satisfaction. The explanation of the constraint consists of two steps: equation (8) computes the lack of interoperative time between two successive care services requested by one patient while the satisfaction regarding this interoperative delay is calculated by (9).

$$
\begin{aligned}
& \operatorname{timelack}\left(s_{k}, s_{k+1}, p\right)=\operatorname{delay}\left(s_{k}, s_{k+1}, p\right)-\min \left(\operatorname{start}\left(s_{k+1}\right)\right. \\
& -\left(\operatorname{start}\left(s_{k}\right)\right. \\
& \left.\left.+\operatorname{duration}\left(s_{k}\right)\right), \operatorname{delay}\left(s_{k}, s_{k+1}, p\right)\right) \\
& \forall s_{k}, s_{k+1} \in S(p), \forall p \in P \text { and timelack }\left(s_{k}, s_{k+1}, p\right) \\
& \geq 0, \operatorname{delay}\left(s_{k}, s_{k+1}, p\right) \geq 0, \operatorname{start}(s) \geq 0 \\
& \operatorname{satisdelay}\left(s_{k}, s_{k+1}, p\right) \\
& =\frac{1}{1+\left(\frac{100}{r s i\left(s_{k}, s_{k+1}, p\right)}-1\right)\left(\frac{\operatorname{timelack}\left(s_{k}, s_{k+1}, p\right)}{\operatorname{cit}\left(s_{k}, s_{k+1}, p\right)}\right)^{2}} \\
& \forall s_{k}, s_{k+1} \in S(p), \forall p \in P \text { and } \operatorname{satisdelay}\left(s_{k}, s_{k+1}, p\right) \\
& \in[0,1], \operatorname{cit}\left(s_{k}, s_{k+1}, p\right) \geq 0, r \operatorname{si}\left(s_{k}, s_{k+1}, p\right) \\
& \in[0,50]
\end{aligned}
$$

\section{Objective function}

Since all the constraints exist in the components of the equation, in this section, we consider a way to aggregate them to generate our $\mathrm{OF}$ representing all the criteria taken into account. Two parts are included: (i) an integer part to present the feasibility of the requested care services, (ii) a fractional which correspond to the product of the patients' and the caregivers' satisfactions coefficients (stakeholders' satisfaction).

The integer part of the OF is illustrated in (10), the primary objective is to ensure that only feasible care services are available, therefore maximizing this number is the main purpose of the algorithm.

$f_{I}=\sum_{p e \in H} \sum_{s \in S(p e)} \sum_{c \in C} G(s, c)$

The fractional part consists of two components. First, the satisfaction function measurement of caregivers, which contains the aggregation of all components (equations $(1)(2)(3)(4))$. The product of difficulty(c,pe) and $\operatorname{satiscg}(c, p e)$ represent the coefficient for each caregiver.

$f_{F C}=\prod_{c \in C} \prod_{p e \in P E(c)} \operatorname{difficulty}(c, p e) \operatorname{satiscg}(c, p e)$ 
Second, the satisfaction function measurement of patient, which contains the aggregation of all components (equations $(5)(6)(7)(8)(9))$ related to the care services that they undergo. The product of satisdelay $\left(s_{k}, s_{k+1}, p\right)$ and $\operatorname{satiscc}(s, p)$ represents the coefficient for each patient.

$f_{F P}=\prod_{p \in P} \prod_{s, s_{k}, s_{k+1} \in S(p)} \operatorname{satisdelay}\left(s_{k}, s_{k+1}, p\right) \operatorname{satiscc}(s, p e)$

To conclude, the OF is obtained by the maximization of the integration of (10) (11) (12):

$\operatorname{Maximize}\left(f_{I}+f_{F C} f_{F P}\right)$

The resolution approach behavior is therefore governed by this function, so we will primarily look for the feasibility of the solutions, then focus on the optimization in a second step. However, the small amplitude of the optimization range (between 0-1) will greatly simplify the work of the metaheuristic approach we have chosen.

\section{RESOLUTION APPROACH}

\section{A. General presentation}

The mathematical formulation we have just explained encourages us to use an algorithm of the metaheuristic: this type of algorithm considers the objective function as a black box, without knowing the details of the calculations. The metaheuristics are general techniques for problem solving by the approximation of an optimal solution [15]. Hence, the method is restarted based on the selected neighborhood solutions. In order not to get blocked in a possible local optimum, the method introduces some randomness allowing it not to take the same direction frequently. These controlled random perturbations are to relaunch the search for the "optimization" by leaving local optimums. Therefore, the algorithm may tend towards excellent solutions in the end.

The metaheuristics have different natures, based on various mechanisms to solve different problems. The most classical metaheuristics in the literature include Genetic Algorithms, Simulated Annealing, Tabu research and Ant Colony optimization. We adopt the Simulated Annealing (SA) as our resolution approach, because of its better performance for Vehicle Routing Problems (VRP) in terms of computational time [16]. In addition its simplicity avoids running out of memory, when faced with our complicated mathematic formulation of the OF and our large-dimension use case to be solved [17]. Furthermore, a comparison study of metaheuristic [18] shows that SA has the better performance in travel cost and computing time by using an empirical delivery data set of Yogyakarte city, in solving delivery routing optimization.

\section{B. Simulated Annealing presentation}

The Fig. 1 shows the functionality of the SA optimization. During each iteration, the SA will keep the best neighbor solution found and compare it to the current solution. If the neighborhood is better, it is automatically retained. Otherwise, the probability which depends on the temperature $T$ will decide the replacement situation of the current solution. The probability we adopt for the neighborhood selection is:

$p=\exp \left(-\frac{\left[f_{O B J}(\text { current solution })-f_{O B J}(\text { best neighbor solution })\right]}{T}\right)$

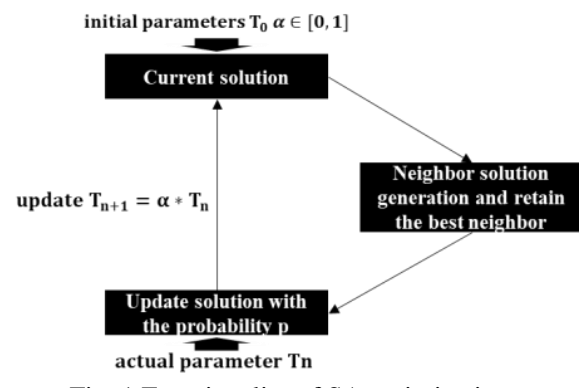

Fig. 1 Functionality of SA optimization.

Different "exchanges" are possible to reverse the order of the care services for generating the neighboring solution. In our case, they are used to change the position of care service in a round, or even to change the round to which a care service is already assigned.

The parameters $\alpha$ and $T_{0}$ therefore directly influence the probability $p$ at each iteration. It is essential to select $T_{0}$ which is large enough to ensure $p \approx 1$ during the first iteration, and $\alpha<1$ allows the decreasing of $T$, but close enough to 1 not to decrease it too quickly after each iteration. $N$ is the number of the neighborhood solutions following the 3 generation rules. Thus, we adopt $T_{0}=1000, \alpha=0.9934, N=30$ as the values for these parameters to generate daily planning in the flow.

\section{NUMERICAL RESULT}

In this section, we introduce one use case to test our daily planning generation considering the satisfaction of both the caregivers and the patients. Then we report the result of this use case by using the simulated annealing based on our mathematic formulation. The algorithm is implemented in Java and the experimental test run on the computer with Intel(R) Core (TM) i7-7500U CPU $2.90 \mathrm{GHz}$ and $16.0 \mathrm{~GB}$ RAM under the Windows 10 operating system.

\section{A. Use case generation}

We generate one instance to test the proposed algorithm with our mathematical formulation. This use case includes 41 patients with a total of 43 care services to be provided. The care services are of 5 different types. Each of the 41 patients have 6 special parameters composing the OF: $r s p=[30,50]$, cts $=$ $[6,15,30,50], \quad r s i=[50]$, cit $=[8,9,30,50]$, delay $=$ $[30,90,150,180]$, gir $=[1,2,3,4,5,6] .10$ caregivers are ready to execute the care service. The caregivers have a total of 5 different skills to accomplish the corresponding care services. Each of the 10 caregivers also have the 2 special parameters to compose the OF: $r s=[50], c t=[6,10,15,20,30,45]$.

\section{B. Experimental result}

We focus on the quantitative studies of our objective function and the performance of algorithm SA with the given algorithm parameter $\left(T_{0}=1000, \alpha=0.9934, N=30\right)$. The algorithm localizes the global optimum when the temperature reaches an extremely low level. We assume approximately 0 degree as the minimum temperature limit. The decrease of temperature from 1000 to approximately 0 need therefore 2882 iterations, with a computational time of 8821 millisecond.

For the feasibility part $f_{I}$, Fig. 2 the shows that in the 246 iterations, SA succeeds in the evolution of the $f_{I}$ and the 43 care services are solved by a feasible solution. Furthermore, the 
curve trend demonstrates that the solution allows the feasibility of increasing care services.

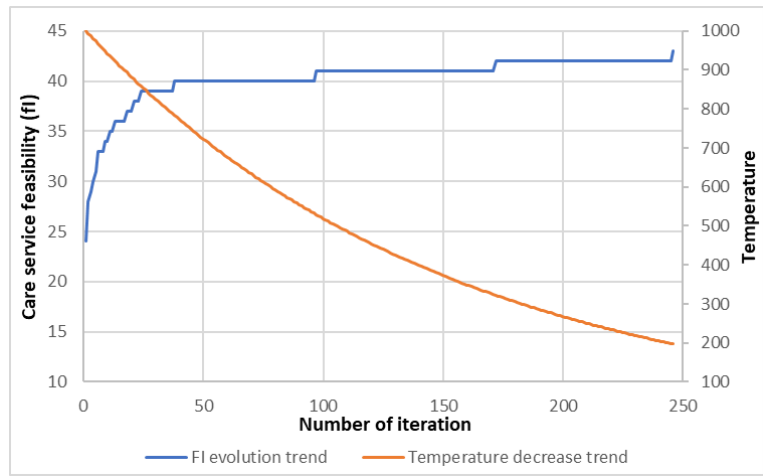

Fig. 2 Evolution of the $\boldsymbol{f}_{\boldsymbol{I}}$ while the temperature decreases

For the optimization part $f_{F C} f_{F P}$, the care services assigned right at the beginning of our optimization $\left(f_{I}=43\right)$, three phases observed in the Fig. 3: (i) high amplitude (246 - 1200 iterations) various options are explored as the temperature decreases. (ii) optimization $(1200-1860$ iterations): beyond that, it is too weak to allow the OF to decrease significantly. (iii) stable (1860 -2882 iterations): from here there is no more decrease of the OF value and the solution, therefore, tends to be as close as the global optimum. The value of this part concerning the final solution is 0.3496 .

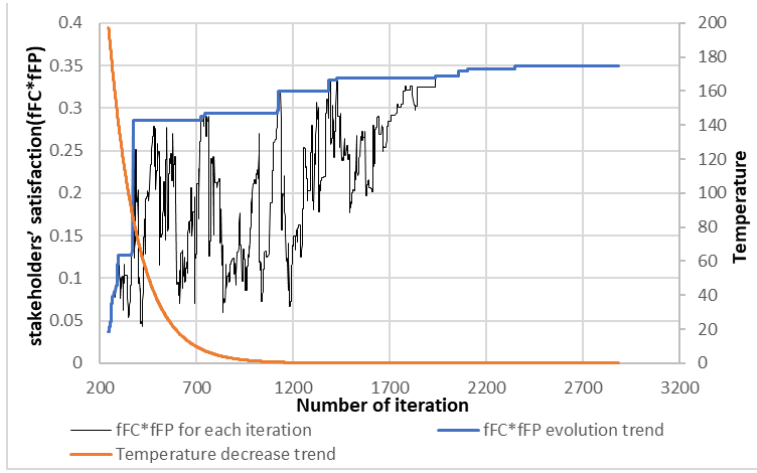

Fig. 3 Evolution of the $\boldsymbol{f}_{F C} \boldsymbol{f}_{F \boldsymbol{P}}$ while the temperature decrease

The result demonstrates the performance of SA in reaching the optimum solution. The final solution is obtained in a reasonable computational time, representing the daily planning with the maximum consideration of all stakeholders' satisfaction based on our mathematical formulation for the metaheuristic.

\section{CONCLUSIONS AND RESEARCH PERSPECTIVES}

This research work addresses the HHCRSP based on the consideration of the overall satisfaction of all the stakeholders. We adopted the metaheuristic-oriented formulation for modelling the problem and the sensibility for controlling the $\mathrm{OF}$ to optimize the solution efficiently. By using the SA algorithm, we generated one use case instance to test our mathematical formulation and to demonstrate the numerical result. During the result display section, a performance analysis of the SA applied to our work aims to determine the optimality of the solution.

In future, we first envision completing this work with a benchmark study. Literature review will allow us to choose suitable benchmarks to test our solution. Then the explanation of uncertainty sources which have an impact on the efficiency of coordination will be taken into consideration. In addition to the techniques of exploiting the optimum in a deterministic situation $\mathrm{o}$, the research will have to make use of uncertainty sources risks for studying the solutions in a non-deterministic situation.

\section{REFERENCES}

[1] E. Lamine et al., "Plas'O'Soins: An Interactive ICT Platform to Support Care Planning and Coordination within Home-Based Care," IRBM, vol. 40, no. 1, pp. 25-37, Feb. 2019.

[2] M. Cissé, "Modèles mathématiques et algorithmes pour la résolution du problème de tournées du personnel de soins à domicile," thesis, Tours, 2017.

[3] S. R. AIT HADDADENE, "Modèles et méthodes pour la gestion logistique optimisée dans le domaine des services et de la santé," thesis, Troyes, 2016.

[4] D. S. Mankowska, F. Meisel, and C. Bierwirth, "The home health care routing and scheduling problem with interdependent services," Health Care Manag Sci, vol. 17, no. 1, pp. 15-30, Mar. 2014.

[5] L. En-nahli, H. Allaoui, and I. Nouaouri, "A Multi-objective Modelling to Human Resource Assignment and Routing Problem for Home Health Care Services," IFAC-PapersOnLine, vol. 48, no. 3, pp. 698-703, Jan. 2015.

[6] A. Trautsamwieser and P. Hirsch, "A Branch-Price-and-Cut approach for solving the medium-term home health care planning problem," Networks, vol. 64 , no. 3, pp. 143-159, Oct. 2014

[7] Z. Yuan and A. Fügenschuh, Home health care scheduling: a case study. Helmut-Schmidt-Univ., Professur für Angewandte Mathematik, 2015.

[8] Y. Shao, J. F. Bard, and A. I. Jarrah, "The therapist routing and scheduling problem," IIE Transactions, vol. 44, no. 10, pp. 868-893, Oct. 2012.

[9] G. Hiermann, M. Prandtstetter, A. Rendl, J. Puchinger, and G. R. Raidl, "Metaheuristics for solving a multimodal home-healthcare scheduling problem," Cent Eur J Oper Res, vol. 23, no. 1, pp. 89-113, Mar. 2015.

[10] R. Redjem and E. Marcon, "Operations management in the home care services: a heuristic for the caregivers' routing problem," Flex Serv Manuf J, vol. 28, no. 1-2, pp. 280-303, Jun. 2016.

[11] G. Du, X. Liang, and C. Sun, "Scheduling Optimization of Home Health Care Service Considering Patients' Priorities and Time Windows," Sustainability, vol. 9, no. 2, p. 253, Feb. 2017.

[12] B. Yuan, R. Liu, and Z. Jiang, "A branch-and-price algorithm for the home health care scheduling and routing problem with stochastic service times and skill requirements," International Journal of Production Research, vol. 53, no. 24, pp. 7450-7464, Dec. 2015.

[13] K.-D. Rest and P. Hirsch, "Daily scheduling of home health care services using time-dependent public transport," Flex Serv Manuf J, vol. 28, no. 3, pp. $495-525$, Sep. 2016

[14] C. Martinez, M. Espinouse, and M. D. Mascolo, "Continuity of care in home services: a client-centered heuristic for the home health care routing and scheduling problem," in 2018 5th International Conference on Control, Decision and Information Technologies (CoDIT), 2018, pp. $1045-1050$

[15] J. DREO, A. PETROWSKI, P. Siarry, and E. Taillard, Métaheuristiques pour l'optimisation difficile. EYROLLES, 2003.

[16] L. Wei, Z. Zhang, D. Zhang, and S. C. H. Leung, "A simulated annealing algorithm for the capacitated vehicle routing problem with twodimensional loading constraints," European Journal of Operational Research, vol. 265, no. 3, pp. 843-859, Mar. 2018.

[17] D. Delahaye, S. Chaimatanan, and M. Mongeau, "Simulated Annealing: From Basics to Applications," in Handbook of Metaheuristics, vol. 272, M. Gendreau and J.-Y. Potvin, Eds. Cham: Springer International Publishing, 2019, pp. 1-35.

[18] A. M. S. Asih, B. M. Sopha, and G. Kriptaniadewa, "Comparison study of metaheuristics: Empirical application of delivery problems," International Journal of Engineering Business Management, vol. 9, p. 184797901774360, Jan. 2017. 